\title{
Stereo-DIC uncertainty quantification based on simulated images
}

\author{
R. Balcaen • P. L. Reu • P. Lava • D. Debruyne
}

Received: date / Accepted: date

\begin{abstract}
Stereo digital image correlation (stereo-DIC) is in wide-spread use for full-field shape, motion and deformation-measurements. However there are very few papers investigating the influence of the setup on the measurement uncertainty. This is mainly due to the highly non-linear measurement chain involving both optical and numerical aspects, making it difficult to investigate how error sources are propagated through the stereo-DIC chain. Indeed, it is impossible to separate all the error sources that are present during a physical measurement. This paper tries to investigate a selection of error sources that are present during experiments. This is based on a simulator introduced in a previous article [1] and briefly reviewed here. Based on these simulations we suggest some "best-practices" guidelines of optimal stereo-DIC setups.
\end{abstract}

Keywords Digital image correlation · Uncertainty quantification · Error assessment · Optical techniques

R. Balcaen · D.Debruyne

KU Leuven, Department of Materials Technology, Campus Ghent, Gebroeders Desmetstraat 1, 9000 Ghent, Belgium

E-mail: ruben.balcaen@kuleuven.be

P. Lava

MatchID, Wijmenstraat 21T, 9000 Ghent, Belgium

P. L Reu

Sandia National Laboratories, Albuquerque, New Mexico, United States 


\section{Introduction}

The uncertainty quantification of a measurement technique is of utmost importance; indeed, one wants to know the signal-to-noise ratio in order to asses the quality of a measurement. Obtaining the uncertainty is quite cumbersome with DIC since the measurement involves both optical and numerical components. There are numerous publications when it comes to uncertainty quantification for 2D-DIC (e.g. [2], [3], [4], [5], [6]) since there is no influence of the calibration-procedure, cross-correlation and triangulation, thus making 2D simulations simpler. Unfortunatly, little research has been done regarding uncertainty quantification for stereoDIC (e.g. [7], [8], [9]). In particular the influence of the setup (e.g. camera, focal length of lenses, stereo angle, etc.) on the measurement uncertainty is not yet fully understood. This is unfortunate as stereo-DIC has important advantages, including non-planar specimens and computation of out-of-plane motion to name just two. We address the lack of uncertainty guidelines for stereo-DIC by using a simulator presented in [1] that allows us to isolate various error sources in order to study them and thus isolating the systematic errors from the random errors.

The basic working principle of the FE based image generator is introduced in section 2, with the main elements of the image generator being discussed. For more information we refer the reader to [1]. Please note that great care is taken when generating the images used in this publication and that, as far as the authors know, no bias is introduced by the image generation. Section 3 continues by showing how the systematic errors of a stereo-DIC measurement are influenced by the chosen setup. This section first introduces the adopted simulation methodology and images are generated in order to detect the full-field uncertainty (section 3.1) and the results are compared to the theoretically predicted uncertainty in an ideal case (by using perfect calibration data). Next, in section 3.1.3 imperfect calibrationdata are introduced into the system and the influence of this on the shape of the uncertainty field is investigated. An analogy to experimental data presented 
in [10] is also made to confirm the simulated data. This is followed by analysing how the stereo-angle and the chosen lenses will influence the uncertainty (section 3.2 ). The influence of an under-matched shape function during cross-correlation is explained in section 4 . In section 5 the effect of aliasing is investigated. The paper ends with some guidelines that can help the reader reduce the systematic errors in a stereo-DIC setup. Please also note that all the data was analysed with a subset based stereo-DIC code, the conclusions are consequentially only applicable to local codes. Global stereo-DIC codes were not used and no conclusions can be made for them.

\section{FE based image generator}

The images created for this paper were made with the FE-based image generator presented in [1]. Here we only briefly introduce the method to assist the reader.

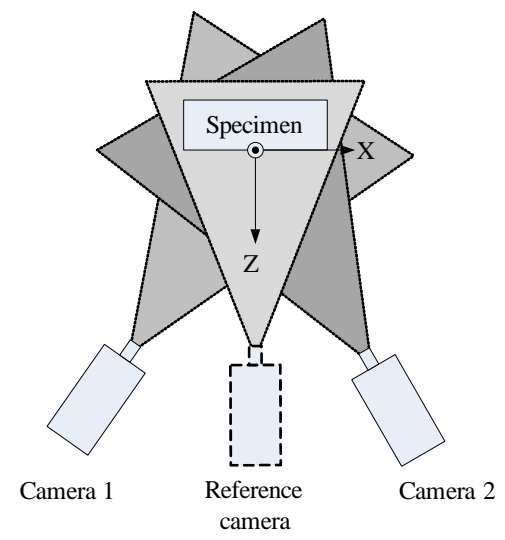

Fig. 1: Camera setup

In order to create deformed images, one needs a reference image (taken during a real test or generated by dedicated software e.g. [11], a reference camera location from where this image was taken (from now on called "the virtual reference camera") and a mesh, representing the object that will be deformed. The mesh is extracted from an FE-package and will be projected 3 times using the pinhole 
camera model [12] as shown in figure 1. This projection will transform points in $3 \mathrm{D}$ space on to $2 \mathrm{D}$ points on the sensor plane of a camera, including lens distortions (both radial as tangent distortions are incorporated [12]). The first projection transforms the FE mesh onto the reference image, as seen from the reference camera. The other two projections transform the same mesh (if no deformation) or a deformed mesh (representing the deformed state) onto a virtual camera image, as seen from the two stereo-cameras in the specified setup. The projections of these meshes are merely a change of the nodal positions of the mesh due to the fact that the cameras are at different positions (thus changing the sensor-location of the projected mesh). No deformation is imposed so far. This is followed by the generation of the deformed image based on the mesh, the reference image and the principles of finite element mapping. The mapping, based on Lagrange polynomials, can be described by the following form:

$$
d=\sum_{i=1}^{N} \Phi_{i} \delta_{a} \hat{u}_{a}
$$

Where $\mathrm{d}$ is the displacement, $\Phi$ is the shape function of the used basis, $\delta_{a}$ is the displacement in direction $a$, where $\hat{u}_{a}$ is the unit vector in direction $a$. A common element type is the linear Q4 element, a bilinear quadrilateral element, based on two Lagrange polynomials. The displacements in this element can be described by 2 local coordinates $[\xi, \eta]$ in a square master-element, where $[\xi, \eta] \epsilon[-1 . .1]$. If one has the global coordinates of the four nodes and the local coordinates of a point, one can determine the global coordinate of that point by mapping it using eq. 1 .

The deforming process calculates the grey-value of each pixel of the deformed image by calculating the grey-value from the reference image, based on the element deformation. The use of the back- and forth-mapping has the advantage that possible interpolation errors in this stage cancel. The main error source is the interpolation of the reference image. If high accuracy is needed, a high-resolution image can be used in order to minimize this error as stated in [13]. This is in 
contrast to the methods used so far for deforming images for a 2D DIC-setup [14], where 3 interpolations were performed. In order to deform images based on an FE-mesh a check must be performed to be sure that the location of the considered pixel is inside an element of the (projected) deformed mesh (denoted as element e). The requirement for this is that the local coordinates $\left[\xi_{g}^{e}, \eta_{g}^{e}\right] \epsilon[-1 . .1]$, where e represents the element and g denotes the fact that the element is in its deformed state. In order to check this requirement the local coordinates have to be calculated from a given global coordinate (the pixel location). This is possible for a standard Q4-element (as described in [15]) by using a different, generic, approach to approximate the inverse mapping with an iterative, updating Taylor expansion as described in [16] and [1].

Since a Taylor-expansion is used, estimated local coordinates are obtained. In order to improve the accuracy of the obtained local coordinate a Gauss-Newton algorithm is used to iteratively optimize $\xi$ and $\eta$. $\xi_{0}$ is updated by $\xi_{i}$ and $\eta_{0}$ is updated by $\eta_{i}$ in the above equations for calculating $\triangle \xi$ and $\triangle \eta$ until the convergence criterion $(\triangle \xi, \triangle \eta) \leq 0.01$ is reached. These mapping functions (from global to local and from local to global) will be used to deform the images; once the local coordinates and the matching element (in its deformed state) are obtained, the global coordinates of this element in its undeformed state can be determined. The authors refer to [16] for more information regarding the mapping. The grey value of the global coordinate in the (interpolated) reference image is then given to the current pixel in the deformed image. A deformed image can thus be created by performing this procedure for all pixels.

After the image generation, several effects can be imposed on the image including lighting effects (i.e. highlights due to reflections), depth of field and motion blur. For more information the reader is directed to the original publication. 


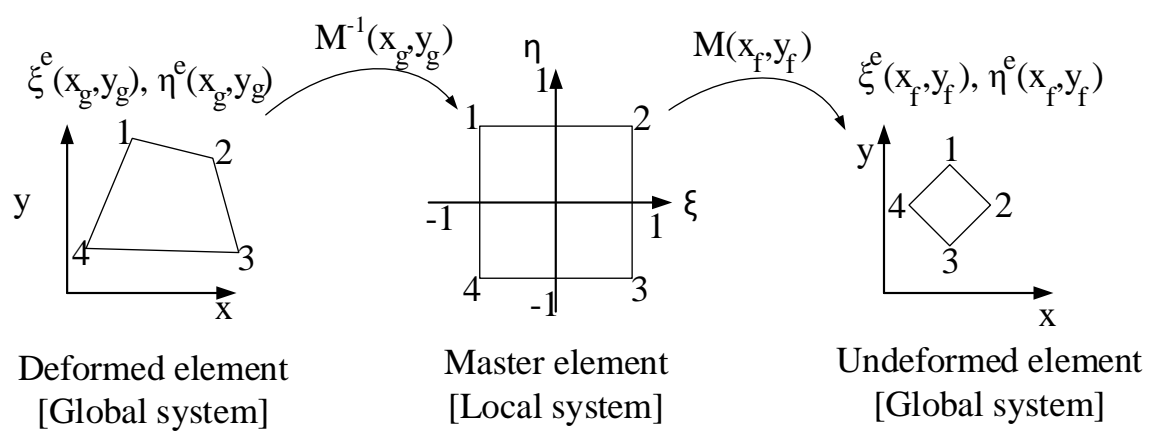

Fig. 2: Transformation between coordinate systems

\section{Influence of experimental setup on accuracy}

This section covers the consequence of the stereo-rig setup on the precision. We include the stereo angle, the focal length of the lenses and the camera noiselevel. These will all have a consequence on the precision of the measurement. Measurement precision in this paper is defined as the standard deviation of the calculated error (difference between the true value and following simulated results). The aforementioned items, which are either known by experience or investigated with time-consuming experiments, are now checked using the simulator. This enables us to exclude the influence of confounding error sources that could cloud our insights. Section 3.1 covers the uncertainty distribution over the FOV, while section 3.2 investigates the influence of different stereo-angles and focal length lenses. The reference pattern used for all the simulations was an 8-bit image of a printed pattern, obtained during an actual experiment. This reference image has good contrast and all speckles (of about 9 pixels per speckle) are evenly distributed. Also even lighting was present, thus no highlights are present in the reference image.

The histogram of the reference image can be seen in figure 3 . 


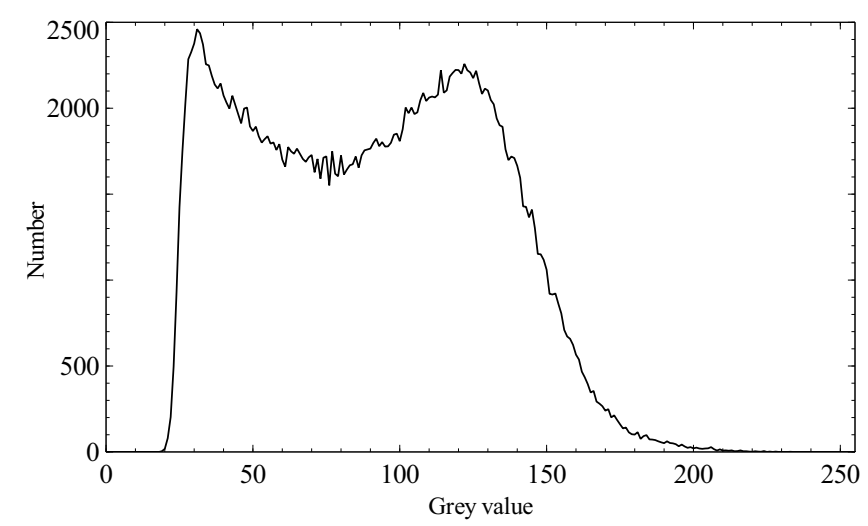

Fig. 3: Histogram reference image

3.1 Full-field standard deviation error plots

The images from both cameras at an identical load step are compared to each other in stereo-DIC in the pursuit of calculating a 3D shape. Indeed; a 2D-DIC run is performed between cameras in order to know the corresponding sensor locations so a triangulation can be executed. There is however an uncertainty on these matched pixel-locations since they are acquired through a 2D-DIC correlation and this results in an uncertainty-region in the triangulated points. The shape of this uncertainty-region depends on the setup and the sensor-matching error as can be seen in figure 4 . The precision in the $\mathrm{x}$-direction will change towards the edge of the FOV since the width of the kite-shape error region will increase (thus changing the predicted uncertainty $[8,17])$, while keeping the uncertainty in the Z-direction (depth) at a constant level. This assumes that the sensor matching error is constant over the entire FOV. This effect is investigated by using simulated images, error propagation theory and a qualitative comparison of simulated data with images from a previous paper. 

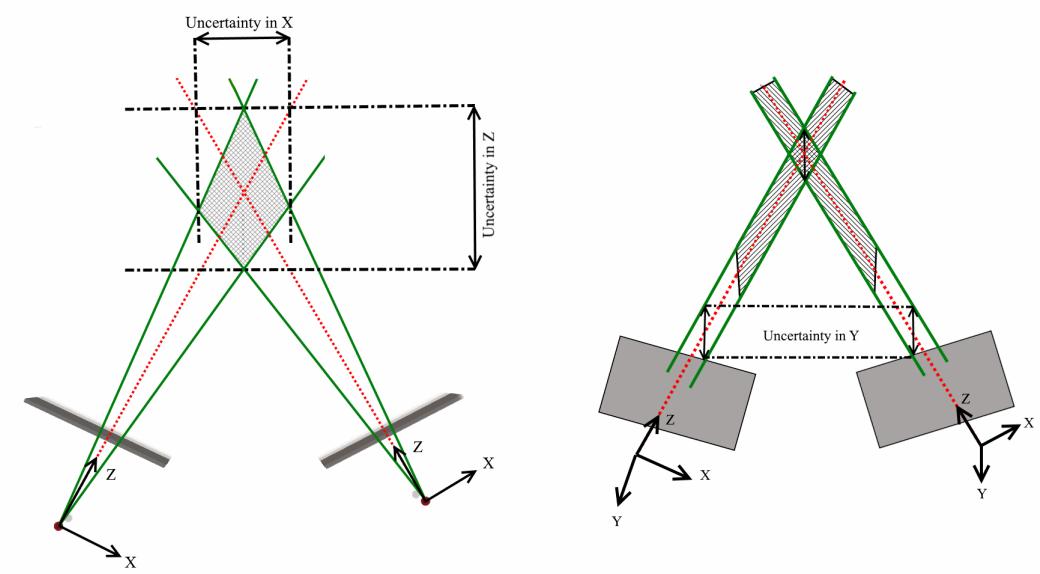

Fig. 4: The best possible precision is determined by the setup and the sensor matching errors $[10]$

\subsubsection{Perfect calibration-data}

In this subsection the shape-reconstruction of a flat plate is simulated with a setup where no lens-distortions are present and where perfect calibration-data is available. This is done for a set of cameras that have $0 \%$ to $2.5 \%$ image noise (with steps of $0.25 \%$ ), and that are set up as a virtual horizontal rig with only rotation around the y-axis $\left(30^{\circ}\right)$ and with lenses with a focal length of $30 \mathrm{~mm}$. If the imposed shape is compared to the measured shape (the images were analysed with MatchID-stereo [18] and with the settings indicated in table 1) the full-field systematic error can be determined. For each DIC-data point the imposed Xcoordinate (defined as $X_{\text {imposed }}$ ) is subtracted from the measured X-coordinate (defined as $X_{\text {measured }}$ ) and this is defined as $X_{\text {error }}$. The same calculation is also used for the Y- and Z-coordinate. The results can be seen in figure 5 (image noise is excluded for this analysis and perfect calibration data is used). It is noticeable that the uncertainty rises near the edges of the FOV (as can be expected from figure 4), but only in the direction of the horizontal setup (X-direction in the case of a horizontal setup); indeed, in the other directions the uncertainty is nearly constant over the FOV. It is clear that the errors in the X-direction are lower, as 
compared to the ones in Y-direction. This can be explained with the conclusions found in section 3.2; the focal length of the lens is beneficial to the precision in the direction of the setup. A higher focal length lens yields lower errors in the direction of the baseline. The baseline was horizontal in this setup, thus the reducing the errors in the X-direction. It is also clear that the errors in the Z-direction are much higher compared to the errors in the X-direction, which can be explained by figure 4 because the sensor matching error creates a kite-shape error field, that always has a bigger height (i.e. the Z-direction) than width (i.e. the X-and Y-direction). The shape of the full-field error does not change if more noise is added, only the standard deviation only increases. This can be seen in figure 6 .

Table 1: Adopted MatchID DIC-settings

\begin{tabular}{lr}
\hline Parameter & Value \\
\hline Camera resolution [pixels] & $1624 \times 1234$ \\
\hline mm per pixel & \pm 0.15 \\
\hline Subset size [pixels] & 21 \\
\hline Step [pixels] & 10 \\
\hline Interpolation & B-spline \\
\hline Shape function & Quadratic \\
\hline
\end{tabular}

\subsubsection{Theoretical uncertainty}

The full-field uncertainty can also be determined in a mathematical way by applying the "error propagation law", in which the triangulation-stage is considered as a black-box system $([19,20])$. If the input covariance matrix (of the matched pixellocations in the image-pairs) and the Jacobian matrix of the system are known the covariance matrix of the output (i.e. the obtained 3D positions) can be calculated as:

$$
C_{y}=F_{x} C_{x} F_{x}^{T}, F_{x i j}=\frac{\partial f_{i}}{\partial X_{c j}}
$$



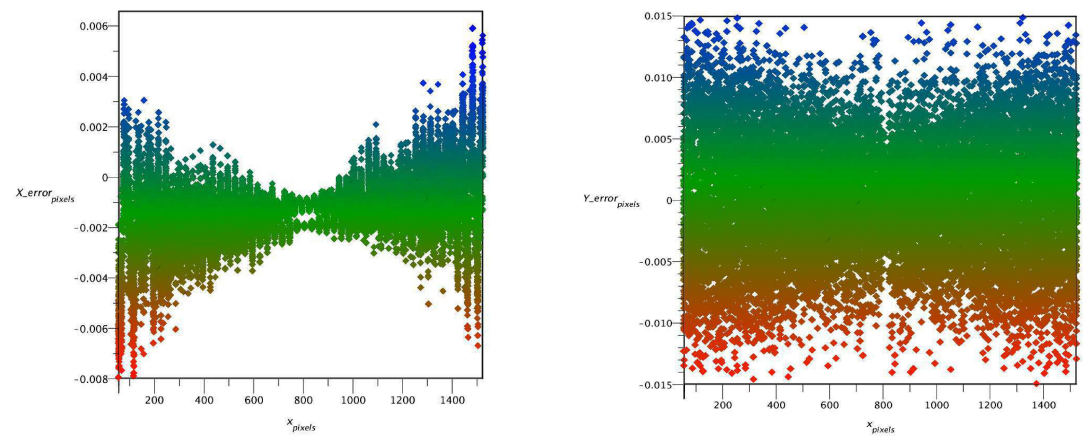

(a) Full-field X-error-perfect calibration (b) Full-field Y-error-perfect calibration data

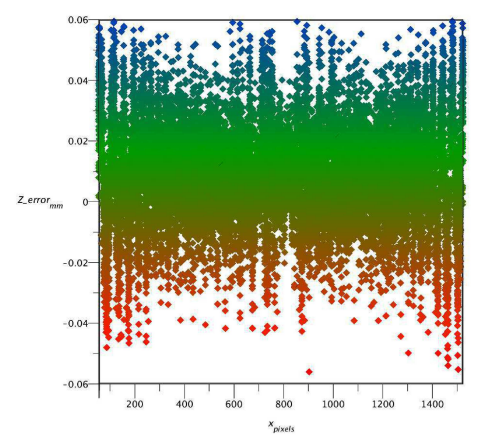

(c) Full-field Z-error-perfect calibration data

Fig. 5: Full-field standard deviation in X-, Y-, and Z-direction

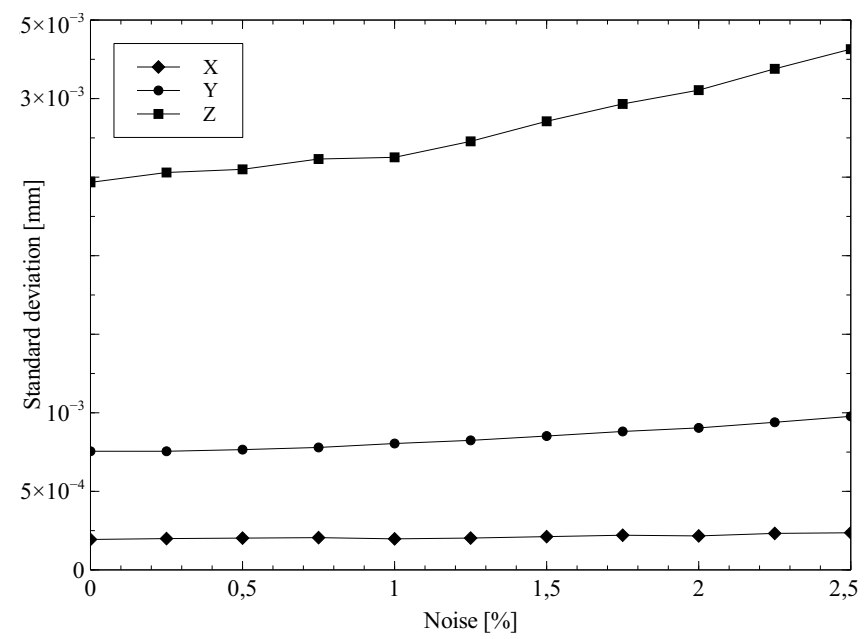

Fig. 6: Influence of image noise on the precision (perfect calibration data used) 
In which $C_{y}$ is the output covariance matrix of the $3 \mathrm{D}$ positions, $F_{x}$ is the Jacobian matrix of the obtained $3 \mathrm{D}$ positions with respect to the matched sensor locations (denoted as $x_{c 1}, y_{c 1}, x_{c 2}, y_{c 2}$ ) and where $C_{x}$ is the input covariance matrix of the same matched sensor locations. This black-box approach can be applied to a generic triangulation procedure (as done in $[19,21]$ ), or specific for stereo-DIC in [7]. The results from [7] are verified by performing the virtual experiment described in section 3.1.1 with the DIC-settings given in table 1 and perfect calibration parameters. In order to determine the covariance of the matched pixellocations from the left to the right camera (denoted as $x_{c 1}, y_{c 1}, x_{c 2}, y_{c 2}$ ) a set of 50 noisy images was generated and the covariance for each matched pixel-pair was determined. The Jacobian was calculated by using a forward finite difference method and the results are presented in figure 7 (markers indicate the mean and the whiskers encompass $99 \%$ of the data) for the uncertainty in the x-direction. It is clear that there is a good correspondence between the theoretical prediction and the simulator-data when good calibration-data is available. Please note that care must be taken when applying this method for real applications; the obtained calibration-parameters will differ from the ideal ones, thus changing the shape and magnitude of the uncertainty-field, as will be shown in the next section.

\subsubsection{Calibration-data with slight error in it}

Since the results from section 3.1.1 are differing from experimentally obtained data (e.g. full-field Y-precision in figure 8 [10]) the possible errors due to erroneous calibration-data were investigated in order to replicate the experimental data. If non-perfect calibration data is used to measure the shape of the plane, a bowlshaped error-plot results as seen in figures 9 (a) to 9 (c). The calibration used to create these error surfaces was created using 50 simulated calibration target images selected at random from a set of 200 images, using the stereo-DIC settings shown in table 2. This indicates that the shape of the full-field uncertainty is mainly dictated by the quality of the calibration. In order to investigate which calibration- 


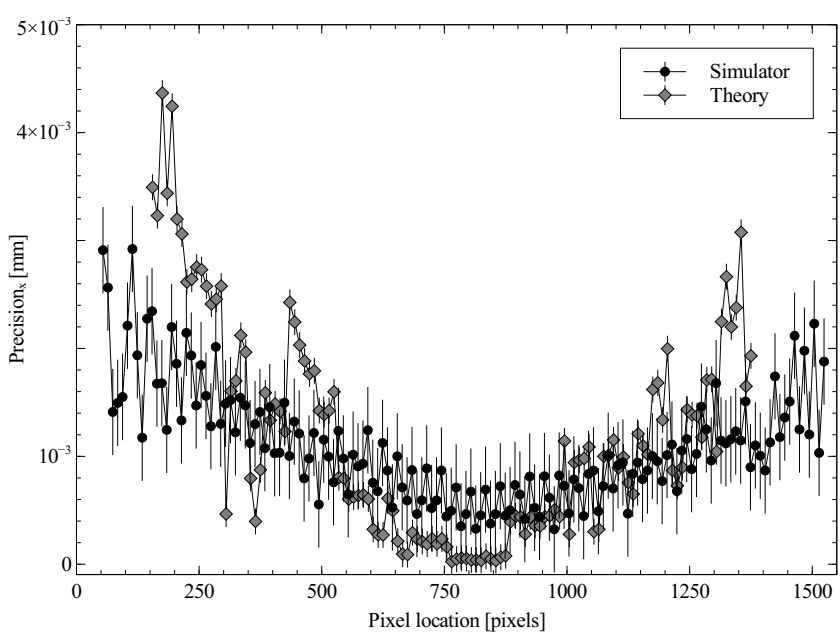

Fig. 7: Theoretically predicted uncertainty versus measured uncertainty by simulated images (perfect calibration data used)

parameters are the driving force a covariance-study with ten different calibrations was performed with the stereo settings given in table 2. The results show that the main error source for the uncertainty in the X-direction is the quality of $C_{x}$ (determining the center of the camera-sensor in a horizontal direction), followed by the tangent distortion factor P2 and the radial distortion factors $\kappa 1, \kappa 2$ and $\kappa 3$ [1]. Similar results are obtained for the precision in the Y-direction; the precision is here mainly determined by the quality of $C_{y}$ (determining the center of sensor in a vertical direction), followed by the tangent distortion factor P1 and the radial distortion factors $\kappa 1, \kappa 2$ and $\kappa 3$. The precision in $\mathrm{Z}$ is mainly determined by the acquired focal length and the measured $T_{x}, T y$ and $T z$ (the relative camera orientations).

\subsubsection{Full-field error conclusions}

One can state that the 3D precision is determined by the sensor matching error in both cameras if perfect calibration data is available. However,the inevitable slight calibration errors will cause a bowl-shaped error-field as shown in figures 8 (experimental data) and $9(\mathrm{a})$ to $9(\mathrm{c})$ (obtained with an imperfect calibration as 


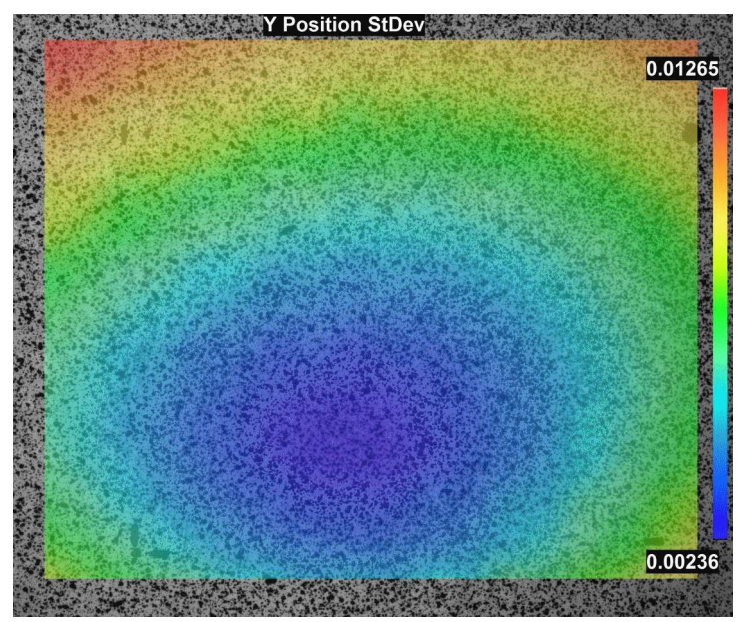

Fig. 8: Experimentally obtained Y-precision [10]

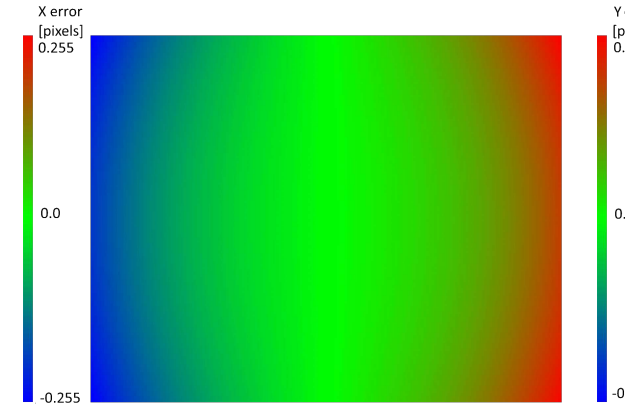

(a) Full-field X-error

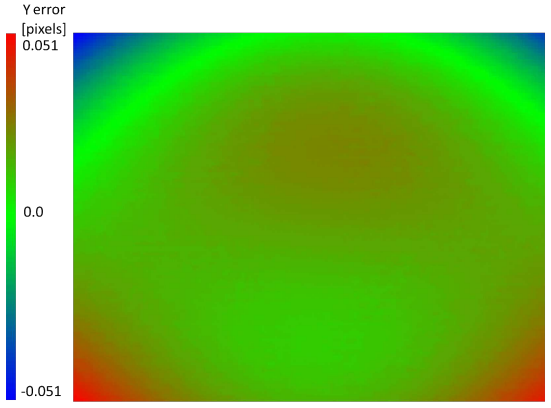

(b) Full-field Y-error

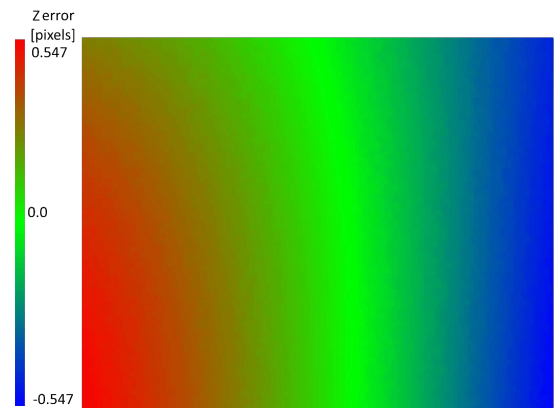

(c) Full-field Z-error

Fig. 9: Full-field precision in X-, Y-, and Z-direction with non-prefect calibration data obtained from calibrating 50 randomly picked images from the set indicated in table 2 
Table 2: Adopted simulated DIC-settings used to create full-filed error plots

\begin{tabular}{lll}
\hline Parameter & Value & \\
\hline Total nr of calibration images & 200 & \\
\hline Images per calibration & 50 & Value Camera 1 \\
\hline Parameter & Value Camera $\mathbf{0}$ & -0.26 \\
\hline$\kappa 1$ & -0.25 & 5.75 \\
\hline$\kappa 2$ & 5.00 & -51.50 \\
\hline$\kappa 3$ & -40.00 & $2.6010^{-4}$ \\
\hline$P_{1}$ & $2.6010^{-4}$ & $1.5510^{-3}$ \\
\hline$P_{2}$ & $1.5510^{-3}$ & \\
\hline
\end{tabular}

discussed previously). Since the errors in the calibration do determine the shape and size of the uncertainty in a measurement, it is of utmost importance to perform a good calibration. It is advised to use the entire FOV during a calibration (thus giving the calibration algorithm better data for modelling the lens distortions since these are mainly of radial nature) and at least 50 good image-pairs must be available for calibration. It is preferred to have more image-pairs, thus enabling the user to remove bad calibration images. The reader is referred to [22] for more information on calibration uncertainty and on how to do a good calibration. Please note that there will always be errors in the calibration data and it is thus advised to mainly use the centre of the FOV since errors will be lower in that region.

3.2 Influence of the focal length and the stereo-angle on the precision

As can be seen in figure 4 the size of the uncertainty-kite is mainly dependent on the stereo-angle of the DIC-setup, the lens focal length and the sensor-error. The sensor error was studied in 3.1, and the influence of the focal length and the stereo-angle will be discussed here. The precision of $\mathrm{Z}$ will improve with a higher stereo-angle, while the errors in of $\mathrm{X}$ and $\mathrm{Y}$ will rise simultaneously, as can be inferred from figure 4. This effect is investigated using the simulator. Images of a flat plate (FOV was about $100 \mathrm{~mm}$ in height and $32 \mathrm{~mm}$ in width) were generated while using different focal length lenses and different stereo angles (summarized in table 3. 
Table 3: Investigated focal lengths and stereo-angles

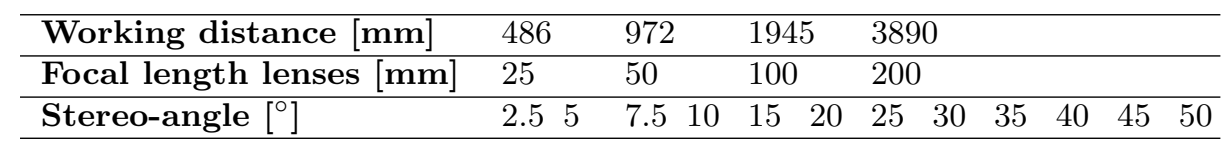

These simulated images were analysed with the MatchID-stereo platform with perfect calibration data generated by the simulator and the measured shape was then compared to the imposed shape (also exported by the simulator). Since the images were generated by a simulator under ideal conditions various error sources could be investigated to determine the effect of the different stereo-angles and focal length of the lenses being used. The following settings were adopted: no image noise, a perfect calibrated system, no camera-motions, perfect flat lighting, a realistic and good pattern that is not aliased and perfect focus over the entire FOV. The results are divided into three sections: 3.2.1, 3.2.2 and 3.2.3, describing different commonly used setups.

\subsubsection{Horizontal setup}

The cameras are placed horizontally next to each other in this section, with only a rotation around the y-axis. Based on figure 4 this would mean that a higher focallength lens yields better results for the X-and Y-direction. However, figures 10(a) to 10 (c) suggest that only the precision in the X-direction (i.e. the direction of the setup) is heavily influenced by the focal-length of the lenses being used. It is also clear that lower errors are present in $\mathrm{Z}$ when a higher stereo-angle setup is used, while a stereo-angle of $15^{\circ}$ or more increases the error in X and Y. Figure 4 explains this geometrically; higher stereo angles will decrease the height of the uncertaintykite (lowering the errors in the Z-direction), while simultaneously gaining width (explaining the higher errors in $\mathrm{X}$ and $\mathrm{Y}$ ). Please also note that the uncertainty in the X-direction (the direction of the setup) is lower than the uncertainty in the Y-direction, regardless of focal length lens. 


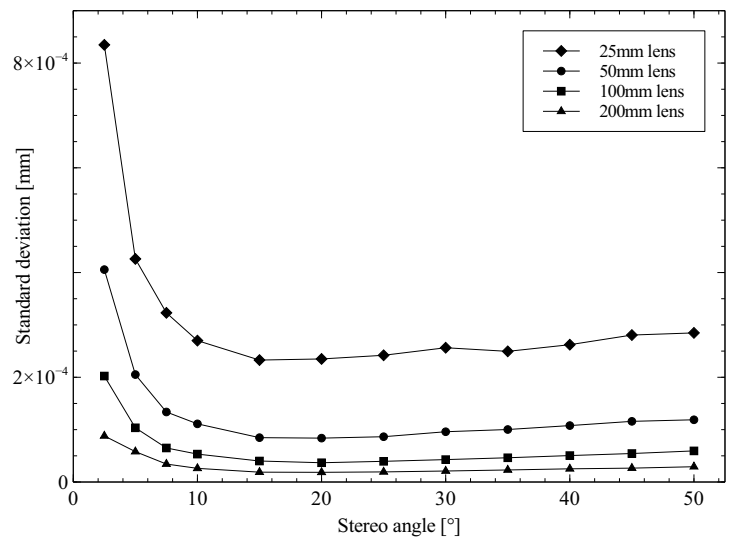

(a) Precision in $\mathrm{X}$-direction

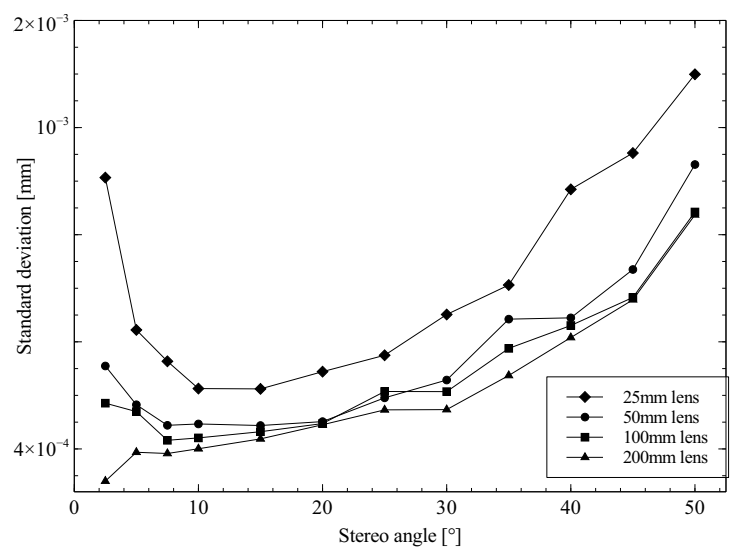

(b) Precision in Y-direction

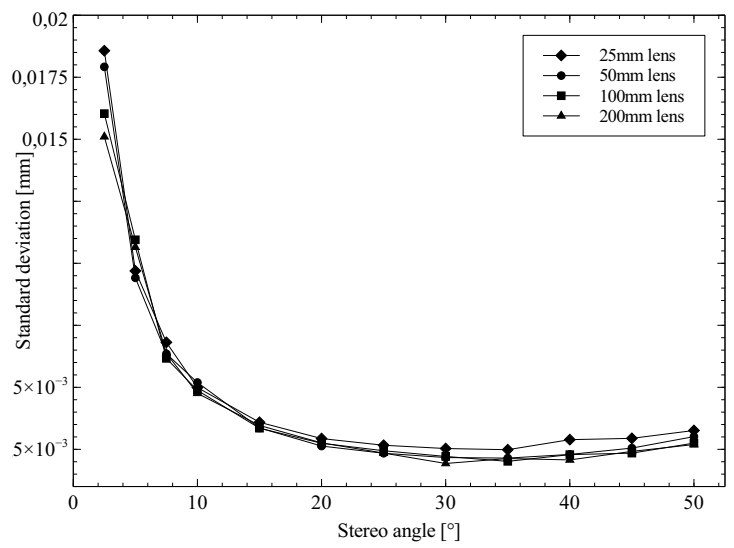

(c) Precision in Z-direction

Fig. 10: Precision in $\mathrm{X}-$, $\mathrm{Y}-$, and Z-direction in function of the stereo-angle and the focal length of the lenses being used, horizontal setup 


\subsubsection{Vertical setup}

An analogous setup as in 3.2.1 is investigated in this section; the setup is however rotated over an angle of $90^{\circ}$. This means that both cameras are positioned above each other and that there is only a rotation around the X-axis. Figures $11(\mathrm{a})$ to 11(c) show that analogous results are obtained as in section 3.2.1. The Z error follows the same trend as in section 3.2.1, while the errors in the $\mathrm{X}$ and $\mathrm{Y}$ follows the same rules.

\subsubsection{Setup with more typical calibration angles}

Since a perfect setup as described in section 3.2.1 or section 3.2.2, with only rotation around one axis to make the stereo angle and no rotation around the other axis, a more realistic setup is simulated in order to validate the results stated in those sections. The simulated setup resembles the horizontal setup (i.e. the baseline of the setup is horizontal), however now with rotations around all 3 axis (the main stereo angles and focal lengths investigated can be seen in table 3 , the rotation around the $\mathrm{x}$-axis was $1.5^{\circ}$ and the rotation around the $\mathrm{z}$-axis was set to $0.08^{\circ}$ ). The angles around the $\mathrm{x}$ - and $\mathrm{z}$-axis were determined from a calibration file from an experiment previously performed by one of the authors. The addition of small rotations about the minor Z-axes yielded similar results as shown in figures 10(a) to $10(\mathrm{c})$. Therefore the results are omitted.

\subsubsection{General conclusion effect of stereo angle and focal length on precision}

As can be seen from figures 10 and 11 small stereo-angles (up to about $10^{\circ}$ ) are unfavourable for the precision in all directions. After this minimum the uncertainties in the in-plane directions rise, while the uncertainty in the depth-direction drops. This effect was also mentioned in [21] (investigating the uncertainty of a generic triangulation-procedure). The reconstruction error in the depth-direction is inversely proportional to the baseline-distance and the related stereo-angle. The 


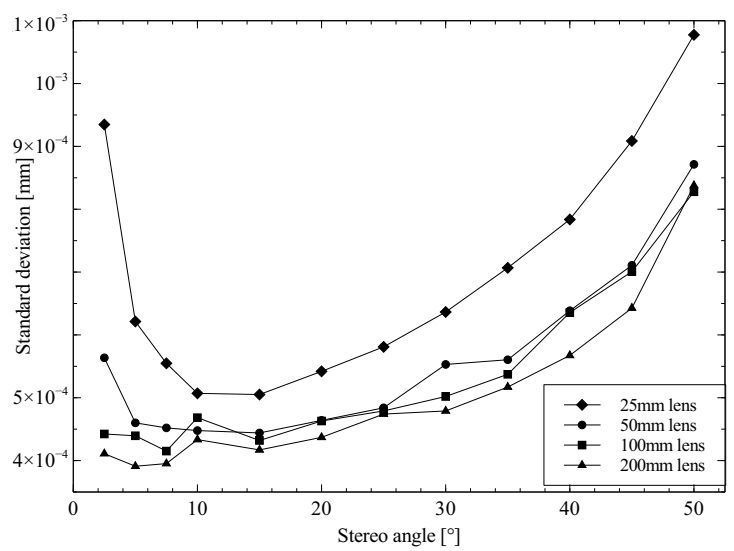

(a) Precision in X-direction

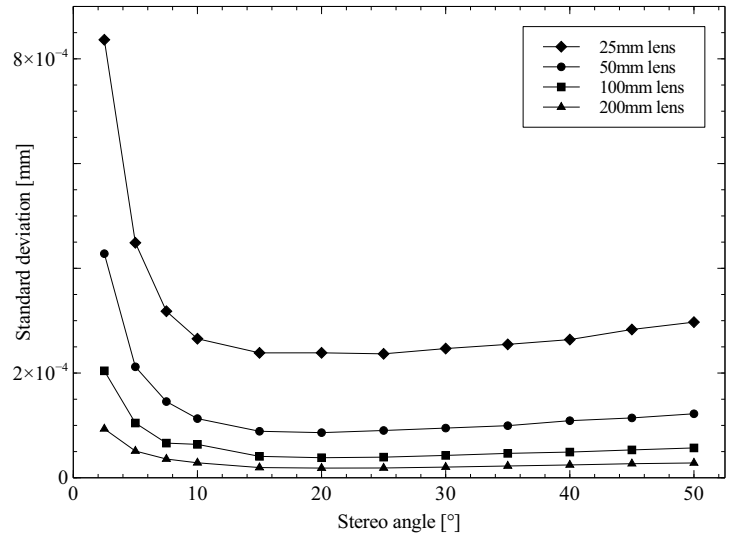

(b) Precision in Y-direction

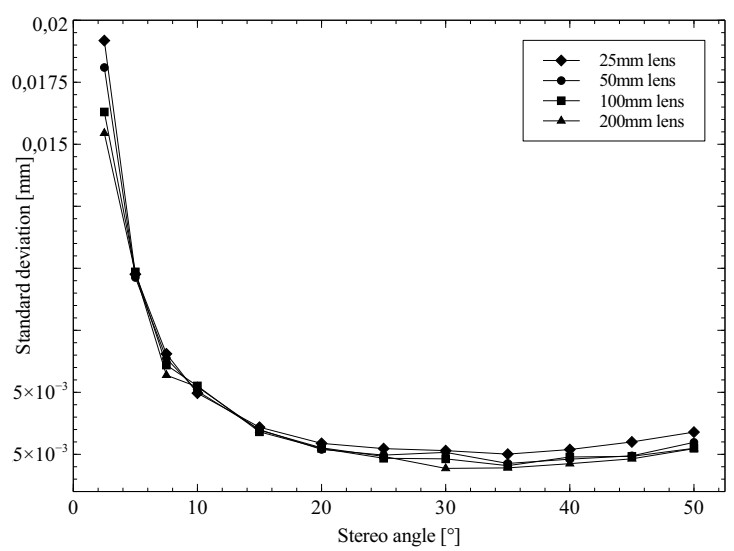

(c) Precision in Z-direction

Fig. 11: Precision in X-, Y-, and Z-direction in function of the stereo-angle and the focal length of the lenses being used, vertical setup 
stereo-angle is proportional to the baseline-distance because the specimen must remain in the FOV of both cameras. The focal length of the lens only influences the $\mathrm{X}$ and $\mathrm{Y}$ errors.

\section{Bias error due to under-matched shape functions in stereo cross-correlation}

In stereo-DIC a cross-correlation is performed between the images from both cameras in order to conduct a triangulation between the matched pixels. This crosscorrelation is a $2 \mathrm{D}$-DIC measurement in which the image from one camera is the reference image and in which the image from the other camera is the deformed image. During this cross-correlation the perspective transformation from one camera to the other is matched by a shape function defined by the DIC-software. This shape-function is usually an affine shape function, which is not completely able to fit the perspective transformation that is present. Consequentially a bias error results in the cross-correlation, as demonstrated earlier in [12]. A possible solution to this problem is using a higher order shape function that is capable of following this perspective transformation (e.g. a projective transformation).

Table 4: Simulation properties cross-correlation bias due to undermatched shape function

\begin{tabular}{lllllll}
\hline Parameter & Value & & & & \\
\hline Camera resolution [pixels] & 1624 x1234 & & & & \\
\hline Focal length lenses [mm] & 100 & & & & & \\
\hline Image noise [counts] & 0 & & & & & \\
\hline Stereo angle system [deg] & 10 & 15 & 20 & 25 & 30 & 35 \\
\hline Subset sizes affine shape function [pixels] & 21 & 31 & 41 & 51 & 101 & \\
\hline Subset size quadratic shape function [pixels] & 41 & 51 & 101 & & & \\
\hline Interpolator & B- & & & & & \\
& spline & & & & \\
\hline
\end{tabular}


In order to validate this a flat plate is simulated that is being imaged with different stereo-angles and the images are analysed with changing subset sizes with an affine shape function. The parameters of the setup can be seen in table 4. The measured cross-correlation displacements are compared to the imposed ones and the difference is marked as the cross-correlation bias. Next, the same experiment is performed with subset sizes of 41,51 and 101 pixels and a quadratic shape function. It is clear from figure 12 that the bias increases with rising subset size and increasing stereo angle if an affine shape function is used. This effect is nearly zero with a quadratic shape function, meaning that this shape function is capable of fitting the perspective transformation. It is thus highly recommended to use a higher-order shape function for the cross-correlation if large subset sizes and high stereo-angles are present. The shape function used for the correlation through time however can stay affine for homogeneous deformation. Please note that the computational cost will rise with a higher-order cross-correlation shape function and that the noise-influence will be slightly higher, as indicated earlier in [23] and [24]. Other solutions for removing this error source are also possible, numerically rectification for example, in which the image is rectified in order to compensate the perspective transformation, but this introduces new error sources (e.g. interpolation errors), making it a less effective solution.

\section{Influence of image aliasing on precision}

Aliased images can often occur during DIC experiments. An aliased image has under-sampled images (a general guideline is to have at least three pixels per speckle in order to avoid this $[12,25])$. The influence of aliasing on the stereo-DIC results is investigated in this section by simulating a uni-axial tensile test on a dog-bone specimen (deformation-field obtained with Abaqus [26]), with the parameters described in table 5 . 


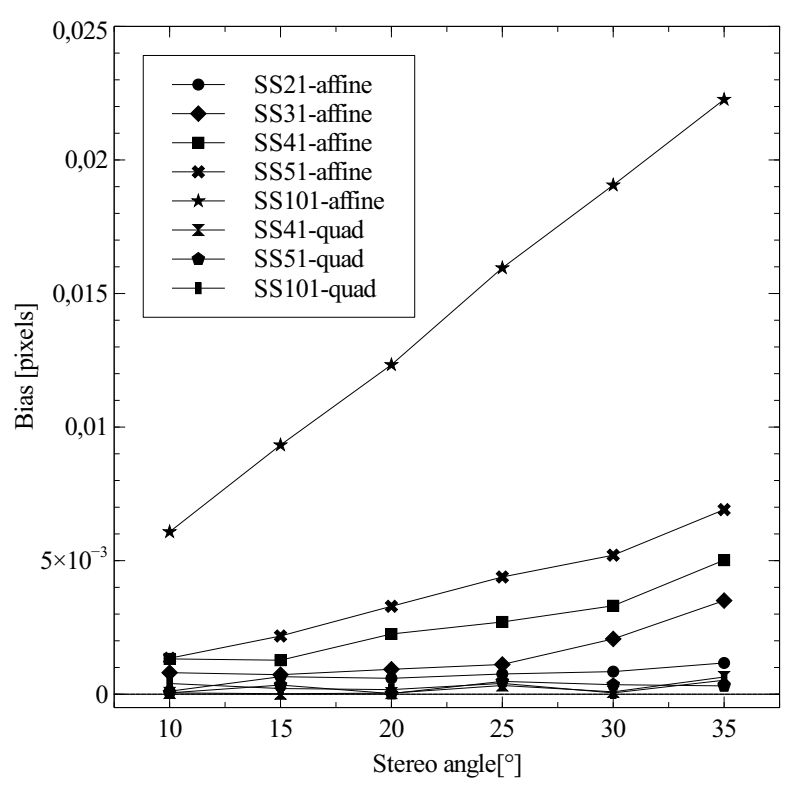

Fig. 12: Cross-correlation bias due to under-matched shape functions

Table 5: Properties of tensile test simulation

\begin{tabular}{ll}
\hline Parameter & Value \\
\hline Specimen width[mm] & 15 \\
\hline Specimen thickness [mm] & 6 \\
\hline Material Young's Modulus [GPa] & 70 \\
\hline Poisson ratio material & 0.30 \\
\hline Element type & C3D8R \\
\hline Number of elements & 18588 \\
\hline Load [N] & 10000 \\
\hline DIC stereo-angle $[\mathrm{deg}]$ & 10 \\
\hline Lenses focal length $[\mathrm{mm}]$ & 50 \\
\hline
\end{tabular}

Two sets of images were generated, one that is aliased (see figure 13(b)) and one that is non-aliased (see figure 13(a)). This is possible with the simulator by changing the position of the virtual reference camera, while keeping the two cameras at their original positions (for more information about the simulator see [1]). By offsetting the virtual reference camera closer to the specimen more pixels will be projected in an element and if the stand-off distance of the cameras to which the element is back-projected to is big enough an aliased image is obtained since 
Table 6: Adopted DIC-settings

\begin{tabular}{ll}
\hline Parameter & Value \\
\hline Camera resolution [pixels] & $1624 \mathrm{x} 1234$ \\
\hline Image noise [counts] & 0 \\
\hline mm per pixel & \pm 0.0787 \\
\hline Subset size "Aliased-modified ss" [pixels] & 9 \\
\hline Step "Aliased-modified ss" [pixels] & 4 \\
\hline Subset size "Aliased" [pixels] & 31 \\
\hline Step "Aliased" [pixels] & 4 \\
\hline Subset size "Non-aliased" [pixels] & 31 \\
\hline Step "Non-aliased" [pixels] & 4 \\
\hline Interpolation & B-spline \\
\hline Transformation & Affine \\
\hline
\end{tabular}

the projection of the same element will be smaller. The pixels of the original image are thus condensed and an aliased image is obtained. The generated images were analysed with MatchID-stereo with perfect calibration data in order to remove unwanted errors. The imposed deformationfield was compared to the calculated one. The DIC settings for the amalysis can be found in table 6 . Note that the aliased images are analysed with two different subset sizes: "Aliased-modified ss" has a smaller subset (chosen according to the "three speckles per subset" guideline), while the "Aliased" results are obtained with the same subset-size as the non-aliased images. Also different interpolators for the DIC-routine were compared to each other. The results can be seen in figures 14(a), 14(c) and 14(e) for shape-measurement and in 14(b), 14(d)and 14(f) for displacement.

The uncertainty in $\mathrm{X}, \mathrm{Y}$ and $\mathrm{Z}$ can rise significantly when an aliased pattern is used as can be seen in figures 14(a) to 14(f). Aliasing should be therefore avoided. To avoid aliasing:

- A higher-resolution camera should be used if there is one available; this will counter-act the aliasing effect and at the same time the spatial resolution will be increased.

- If the specimen does not cover the entire FOV one can move the cameras closer or one can use higher focal length lenses. This increases the speckle-size 

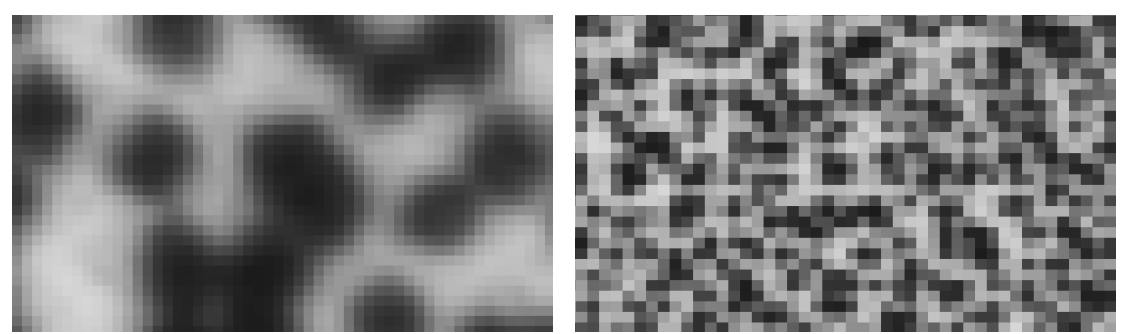

(a) Non-aliased pattern showing well re- (b) Aliased pattern created from the same solved speckles. $\quad$ speckle pattern, but a different relative camera position that makes the speckles too small relative to the stereo-DIC system.

Fig. 13: Different generated patterns for aliasing.

on the sensor, pushing the pattern-size away from the resolution limit of the sensor. Please note that more care must be taken when using the entire FOV since the uncertainty rises near the edge of the FOV because of undermatched lens-distortion factors in the calibration-data.

- One can respeckle the pattern with bigger speckles (if the application allows it).

- If the test is already performed and if the aliasing was not noticed before the effect can be minimized by applying a pre-filter to the image and using a larger subset size, taking the trade-off with decreased spatial resolution into account $[27]$.

It is also clear from figures 14(a) to 14(f) that a bicubic interpolant should be used. A bilinear interpolant can outperform a bicubic one when aliasing is present, mainly when a (too) large subset size is chosen. This is caused by the fact that a bicubic interpolant uses second order derivatives, thus enhancing the aliasing effect. This is not the case for a bilinear interpolant since only the four closest neighbours are used, nor is it the case for a bicubic spline interpolant since the bicubic spline interpolant fits a curve through the given data-points, resulting in a smoothed curve. 


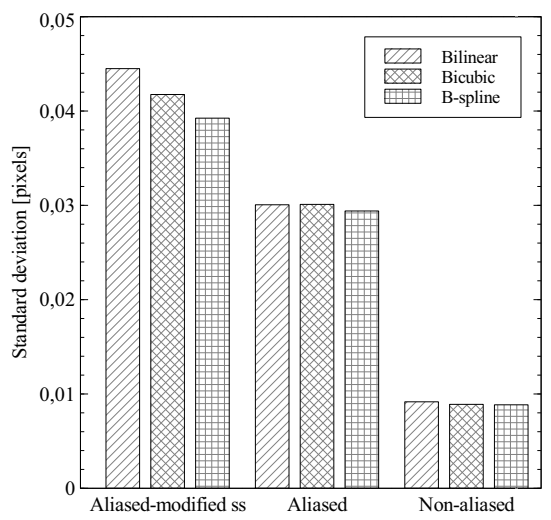

(a) Precision in $\mathrm{X}$

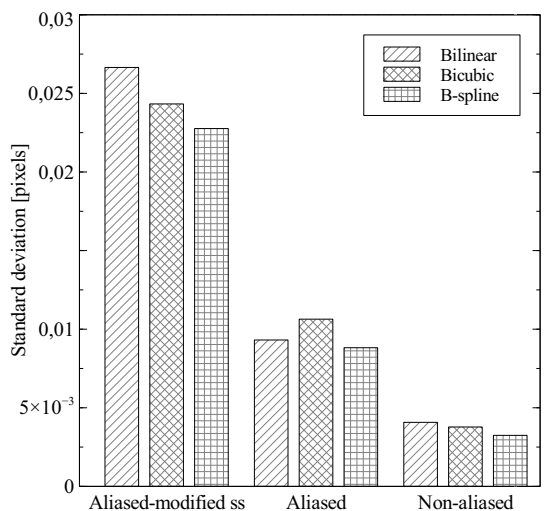

(c) Precision in $\mathrm{Y}$

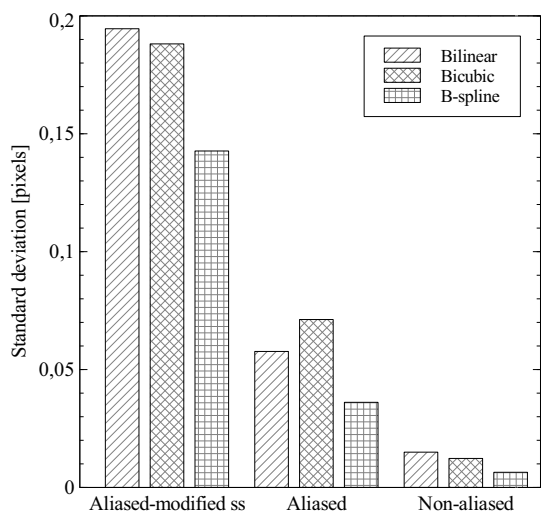

(e) Precision in Z

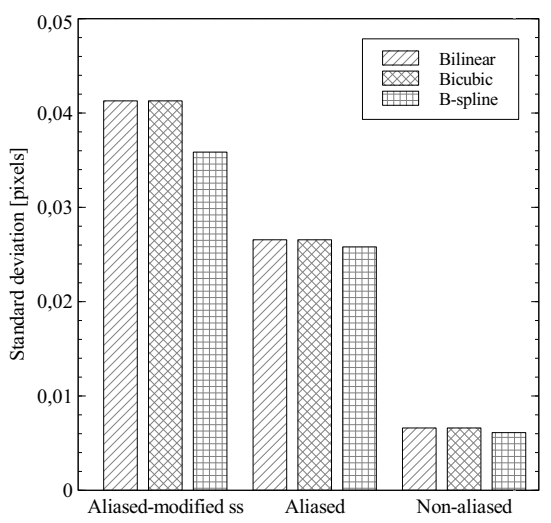

(b) Precision in U

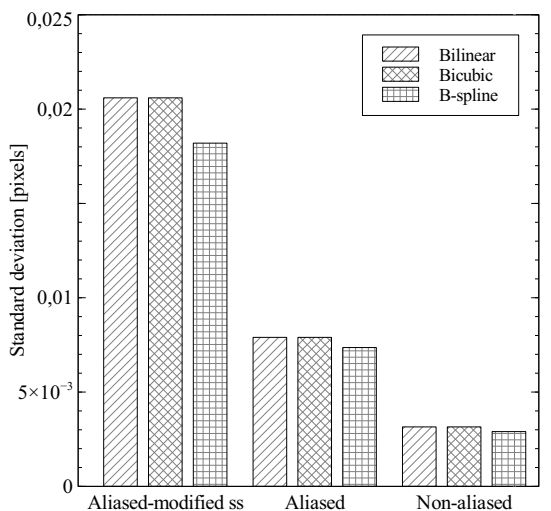

(d) Precision in V

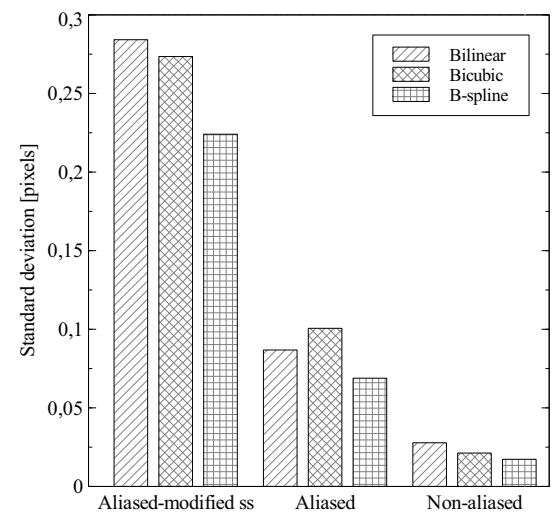

(f) Precision in $\mathrm{W}$

Fig. 14: Influence of aliasing and interpolant on the obtained precision 


\section{Conclusion}

The simulator can be a helpful tool when it comes to uncertainty quantification for stereo-DIC. Various error sources can be introduced and because the imposed deformation-field is exactly known the error can be quantified. Please note that in this paper we only investigated regular two-camera setups. The simulator does offer the possibility of investigating the uncertainty of stereo-DIC in multi-camera setups. We can now use this simulator to investigate many other stereo-DIC situations, including multi-camera DIC and the recently introduced global stereo-DIC $[28]$.

We used the stereo-DIC simulator to validate a large number of existing experimental rules-of-thumb for a DIC course [10], which contains values for the order of magnitude for different error sources present during a stereo-DIC experiment. These values are now verified by means of the simulator (results shown in table 7). The given values are estimates for a $100 \mathrm{~mm}$ FOV with normal lighting, speckle contrast (the pattern is partially displayed in figure 13(a)), image noise and when the images are analysed with the correct correlation parameters. Please note that the mentioned influences are the only ones present and that they represent an order of magnitude, i.e. these are not absolute values, nor can one simply add these

Table 7: Error sources and their magnitude-comparison of [10] and simulator

\begin{tabular}{lll}
\hline Parameter & $\begin{array}{l}\text { Value from DIC- } \\
\text { course [pixels] }\end{array}$ & $\begin{array}{l}\text { Value from sim- } \\
\text { ulator [pixels] }\end{array}$ \\
\hline Cross-correlation & 0.01 & 0.003 \\
\hline Turbulence & 0.02 & Not investigated \\
\hline Camera motion & 0.05 & Not investigated \\
\hline Image noise & 0.01 & 0.01 \\
\hline Speckle size & 0.02 & 0.02 \\
\hline Speckle contrast & 0.01 & 0.005 \\
\hline Aliasing for a normalized speckle & 0.005 & 0.02 \\
\hline Interpolant & 0.001 to 0.01 & $\begin{array}{l}0.001 \text { (no aliasing) } \\
\text { to } 0.01 \text { (aliasing) }\end{array}$ \\
\hline
\end{tabular}


together if multiple error sources are present at the same time.

To prove this statement the same test was simulated with various error sources that were activated in several combinations and a comparison was made with a reference state that didn't include any of the investigated error sources. By comparing to this reference state the influence of other errors (e.g. triangulation errors, numerical errors, interpolation biases, etc.) are removed and the actual order of magnitude of the various errors can be determined. Table 8 illustrates the order of magnitude of the different error sources separately, i.e. only one error source was active at each simulation. These values were obtained when the image noise varied from $0.25 \%$ in the reference state to $1.0 \%$ for the noisy state, the contrast of the image varied from a range of 200 counts to 65 counts (for an 8 bit image) and where a bicubic spline interpolant was used for the reference state versus a bilinear one.

In figure 15 the different error sources are combined by summing the values from table 8 (indicated as "Sum of seperate error values") and by simulating the same test but now when various error sources are present at the same time (indicated as "Simulated superposition of errors"). It is clear that the resulting error is not merely the addition of the different error-values since different error sources can amplify each other or possibly cancel each other out (in the case when a bad interpolant is combined with low speckle contrast).

Table 8: Superposition of error sources

\begin{tabular}{lll}
\hline Parameter & $\begin{array}{l}\text { Magnitude } \\
\text { error [pixels] }\end{array}$ \\
\hline Image noise & 0.015 \\
\hline Speckle contrast & 0.003 \\
\hline Interpolant & 0.006 \\
\hline
\end{tabular}




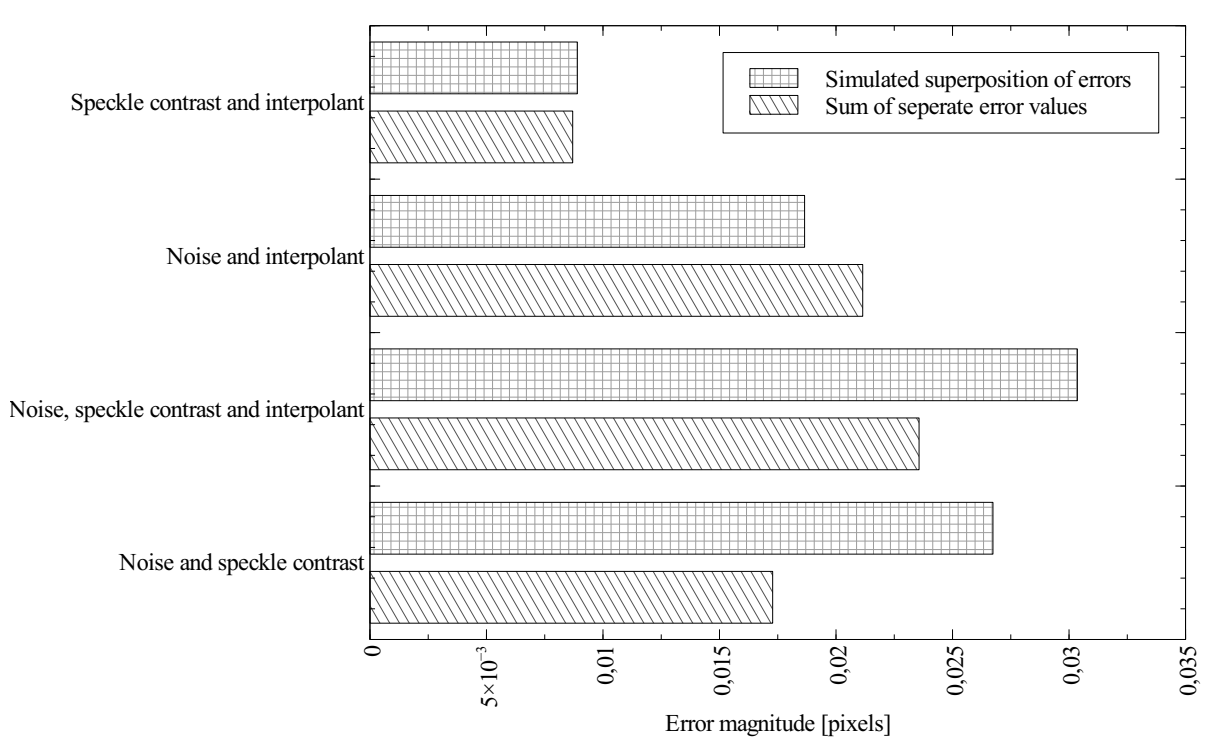

Fig. 15: Error superposition

Another use of the simulator is in designing an ideal stereo-DIC system for a given experiment. Some of these parameters have been studied in this paper. Here we provide some general guidelines that can be used when designing a setup; e.g. by using figure 4 it is possible to get a feeling for the direction with the highest precision. One can geometrically see that a higher stereo angle yields a better precision in the depth-direction, at the cost of the in-plane precision. Higher focallength lenses do not seem to effect the precision in the depth-direction (see figure $10(\mathrm{c})$ and $11(\mathrm{c}))$, while they do seem to slightly improve the precision in the inplane direction of the baseline of the cameras. However, the gain that can be made with optimizing the setup however can be diminished by a bad calibration as can be seen in figures 8 and 9. A good calibration is thus of highest importance in order to fully use the capabilities of the used hardware. The authors refer to the literature (e.g. $[1,22])$ for more information regarding the influence of calibration quality and calibration uncertainty quantification. Also keep the cross-correlation shape function into account; a cross-correlation bias can be present if higher subset-sizes (e.g. 31 and up) and higher stereo-angle are used as can be seen in section 4 . It is 
recommended to switch to a higher order shape function for the cross-correlation matching if needed.

\section{References}

1. R. Balcaen, L. Wittevrongel, P.L. Reu, P. Lava, and D. Debruyne. Stereo-DIC calibration and speckle image generator based on fe formulations. Experimental Mechanics, 57(5):703$718,2017$.

2. M Bornert, F Brmand, P Doumalin, J-C Dupr, M Fazzini, M Grdiac, F Hild, S Mistou, J Molimard, J-J Orteu, L Robert, Y Surrel, P Vacher, and B Wattrisse. Assessment of digital image correlation measurement errors: methodology and results. Experimetal Mechanics, 49(3):353-370, June 2009.

3. Y Q Wang, M A Sutton, H A Bruck, and H W Schreier. Quantitative error assessment in pattern matching: Effects of intensity pattern noise, interpolation, strain and image contrast on motion measurements. Strain, 45(2):160-178, 2009.

4. P Lava, S Cooreman, S Coppieters, M De Strycker, and D Debruyne. Assessment of measuring errors in DIC using deformation fields generated by plastic fea. Optics and Lasers in Engineering, 47(7-8):747-753, 2009.

5. B Pan, K Qian, H Xie, and A Asundi. Two-dimensional digital image correlation for in-plane displacement and strain measurement: a review. Measurement Science and Technology, 20(6):062001, 2009.

6. Phillip L Reu. A realistic error budget for two dimension digital image correlation. Advancement of Optical Methods in Experimental Mechanics, 3:189-193, 2015.

7. YQ Wang, M A Sutton, X D Ke, H W Schreier, P L Reu, and T J Miller. On error assessment in stereo-based deformation measurements-part 1. Experimental mechanics, 51(4):405-422, 2011.

8. X D Ke, H W Schreier, M A Sutton, and Y Q Wang. On error assessment in stereo-based deformation measurements-part 2. Experimental mechanics, 51(4):423-441, 2011.

9. Phillip L. Reu. Imaging methods for novel materials and challenging applications, volume 3. Springer New York, 2013. Conference proceedings of the society for experimental mechanics series 2013 pp 311-317.

10. P. Lava, F. Pierron, and P.L. Reu. Dic course-metrology beyond colors, January 13-16 2014.

11. J. J. Orteu, D Garcia, L Robert, and F Bugarin. A speckle texture image generator. In Proc. SPIE 6341, Speckle06: Speckles, From Grains to Flowers, 63410H, 2006.

12. M. A. Sutton, J.J. Orteu, and H. W. Schreier. Image correlation for shape, motion and deformation measurements. Springer Science, 2009. 
13. Y. Wang, P. Lava, and D. Debruyne. Using super-resolution images to improve the measurement accuracy of DIC. In Optical measurement techniques for systems and structures III, pages 353-361, April 2015.

14. Y Wang, P Lava, S Coppieters, M De Strycker, P Van Houtte, and D Debruyne. Investigation of the uncertainty of DIC under heterogeneous strain states with numerical tests. Strain, 48(6):453-462, 2012.

15. C. Hua. An inverse transformation for quadrilateral isoparametric elements: Analysis and application. Finite Elements in Analysis and Design, 7(2):159-166, November 1990.

16. L. Wittevrongel. A self adaptive algorithm for accurate strain measurements using global digital image correlation. Other titles: "Een zelfcorrigerend algoritme voor nauwkeurige rekmetingen met behulp van globale digitale beeldcorrelatie.”. PhD thesis, KU Leuven, https://lirias.kuleuven.be/handle/123456789/506239, October 272015.

17. P. L. Reu. Stereo-rig design: Stereo-angle selection part 4. Experimental Techniques, $37(2): 1-2,2013$.

18. MatchID. http://www.matchidmbc.com/.

19. J. Chen, Z. Ding, and F. Yuan. Theoretical uncertainty evaluation of stereo reconstruction. In 2nd International Conference on Bioinformatics and Biomedical Engineering, pages 2378 - 2381. IEEE, 2008.

20. M. G. Cox, M. P. Dainton, and P. M. Harris. Software support for metrology best practice guide no. 6 - uncertainty and statistical modelling. Technical report, Centre for Mathematics and Scientific Computing, 2001.

21. G Di Leo, C Liguori, and A Paolillo. Propagation of uncertainty through stereo triangulation. In Instrumentation and Measurement Technology Conference (I2MTC), pages 12-17. IEEE, 2010.

22. P L Reu. A study of the influence of calibration uncertainty on the global uncertainty for digital image correlation using a monte carlo approach. Experimental mechanics, 53(9):1661-1680, 2013.

23. W. Schreier H. and A. Sutton M. Systematic errors in digital image correlation due to undermatched subset shape functions. Experimental Mechanics, 42(3):303-310, 2002.

24. L Yu and Bing Pan. The errors in digital image correlation due to overmatched shape functions. Measurement Science and Technology, 26(4):045202, 2015.

25. P. L. Reu. The art and applications, all about speckle: aliasing. Experimental Techniques, $38(5): 1-3,2014$.

26. Abaqus. http://www.3ds.com/productsservices/simulia/products/abaqus/.

27. P L Reu, W Sweatt, T Miller, and D Fleming. Camera system resolution and its influence on digital image correlation. Experimental mechanics, 55(1):9-25, January 2015. 
28. J Neggers, J P M Hoefnagels, F Hild, S Roux, and M G D Geers. A global digital image correlation enhanced full-field bulge test method. IUTAM Symposium on Full-field Measurements and Identification in Solid Mechanics, 4:73-81, 2012. 